\title{
Wavelet-artificial immune system algorithm applied to voltage disturbance diagnosis in electrical distribution systems
}

ISSN 1751-8687

Received on 4th April 2014

Accepted on 19th December 2014 doi: 10.1049/iet-gtd.2014.1102 www.ietdl.org

\author{
Fernando P.A. Lima, Anna Diva P. Lotufo, Carlos Roberto Minussi $\bowtie$ \\ Electrical Engineering Department, Faculty of Engineering of Ilha Solteira, UNESP, Universidade Estadual Paulista 'Júlio de Mesquita \\ Filho', Av. Brasil 56, P.O. Box 31, Ilha Solteira, SP 15385-000, Brazil \\ $\bowtie$ E-mail: minussi@dee.feis.unesp.br
}

\begin{abstract}
This study presents a new approach to detecting and classifying voltage disturbances in electrical distribution systems based on wavelet transform and artificial immune algorithm. This proposal unifies the negative selection artificial immune algorithm with the discrete wavelet transform concept. Thus, the measurements obtained in a distribution substation by the supervisory control and data acquisition acquisition system are transformed into the wavelet domain. Afterward, a negative selection artificial immune system realises the diagnosis, identifying and classifying the abnormalities. The principal application of this tool is to aid the system operation during faults as well as to supervise the protection system. To evaluate the performance of the proposed method, two distribution systems were modelled in EMTP software: an 84-bus test system and a 134-bus real system. The results show a good performance, emphasising the precision of the diagnosis.
\end{abstract}

\section{Introduction}

Recently, the electrical power companies have been modernising distribution equipment and automating the operations process, which reduces the cost [1]. The investments are intended to satisfy the rules that are imposed by the regulator agencies and to increase the reliability, security and efficiency of the system. To accomplish these goals, two technologies are employed: digital technology and information technology [2]. With these technologies, it is possible to develop integrated systems that combine acquisition, analysis and data processing techniques with control and decision making [3].

Failure diagnosis is commonly based on visual inspection of the oscillograph. Expert operators aid in the decision making. However, they cannot identify every fault, and the process can be inefficient and insecure. Therefore intelligent techniques are a good alternative for solving the diagnosis problem. Several techniques based on this concept are used to aid the operators to execute the routines, providing security, efficiency and velocity to corrective actions. Some of the relevant studies that are available in the literature are described below.

The study in [4] presents a system for recognising voltage disturbances in distribution electrical systems in the wavelet transform domain. The Dempster-Shafer evidence theory [5] is used to integrate the output of a neural network set that classifies the disturbances. The work of [6] presents a new approach to power quality disturbance diagnosis, using a wavelet transform and the Kohonen neural network. In [7], the authors develop and use a voltage disturbance diagnosis based on neural networks, fuzzy logic and wavelets. The study in [8] presents a methodology that is based on wavelets and entropy norms with a perceptron multilayer neural network that is trained by back propagation [9] to classify voltage disturbances. A hybrid system with a wavelet transform and an artificial neural network is used to detect, locate and classify electrical power quality disturbances in [10].

The study in [11] presents a method for detecting and classifying power quality disturbances using the discrete wavelet transform (DWT) and a wavelet neural network. The wavelet transform is used to extract characteristics that will be analysed by the wavelet neural network and to classify the signal.

The work proposed in [12] presents a real-time power quality disturbance classification method. This method used the discrete
Fourier transform to extract signals from a decision tree based on rules (rule-based decision tree) to classify the disturbances. The study in [13] presents a method for performing a voltage disturbance diagnosis in electrical distribution systems. The wavelet transform is used to extract the characteristics from the voltage oscillograph, and the information is joined by the entropy concept into a fuzzy-ARTMAP neural network, which classifies the disturbances.

The study in [14] proposes a new approach to voltage disturbance diagnosis by using a negative selection immune system. This approach uses proper/no proper discrimination to differentiate the signals. The resulting diagnosis system presented excellent results. And in the paper [15] was presented a methodology for optimal database storage of disturbances in electrical distribution systems using artificial immune systems (AISs).

In the specialised literature, there is no publication that is related to voltage disturbances in electrical distribution systems using AISs and wavelet transforms. Therefore the proposal of this work aims to contribute to this area by presenting a new algorithm for voltage disturbance diagnosis that uses AISs and wavelet transforms. The AISs were originally proposed to solve computational security problems, and they produced good results. Owing to the good results in other areas, this work proposes a new approach to performing failure diagnosis in electrical energy systems.

The AISs are promising algorithms in artificial intelligence (AI); the concept is based on biological immune systems (BISs) and aims to computationally reproduce its principal characteristics, properties and abilities [16]. As emphasised in [17], the AIS is adequate tools to be applied in failure diagnosis of electrical systems due to the natural characteristics of diagnoses. These characteristics are related to biological inspiration. The AIS was inspired in the BIS, which have natural characteristics of diagnosing of disease in the human organism.

The wavelet transform is a mathematical tool for signals analysis through decomposition or breakage of the constituent parts, allowing to analyse the data in different levels of frequency with the resolution of each component in its range. In summary, the wavelet transform allows you to view the approximation of the discontinuous data in functions, that is, view the abnormalities in the signals, so becomes an important tool in the analysis and diagnosis of abnormality in the electrical system. The use of a wavelet 
transform provides, to the diagnosis system, a sensitivity that allows the system to easily identify abnormalities in the signals.

Therefore this paper presents a new approach to performing voltage disturbance diagnosis in electrical distribution systems by using a wavelet-AIS. This immune-wavelet proposal aims at robustness and efficiency (precision) in the diagnosis process. To evaluate the performance, tests were conducted with two electrical distribution systems: one system was a test system with 84-bus, and the other system was a real system with 134-bus.

This paper is organised as follows: Section 2 presents the negative selection algorithm (NSA). Section 3 describes the wavelet transform. The modelling and simulation is presented in Section 4. Section 5 presents the proposed methodology. Finally, the results and conclusions are presented, respectively, in Sections 6 and 7.

\section{Negative selection algorithm}

The NSA, which was proposed in [18] to detect changes in systems, is based on the negative selection of T lymphocytes over time. This process works on the discrimination of proper against non-proper cells. The algorithm is executed in two phases, according to the following description $[16,19]$ :

\section{Censor}

(a) Define a set of proper chains (S) to be protected.

(b) Generate random chains and evaluate the affinity (match) between each chain and the proper chains. If the affinity is greater than a predefined value, then reject the chain. Otherwise, file the chain into a detector set (R).

\section{Monitor}

(a) Given a set of chains to be protected (protected chains), evaluate the affinity with each chain and the detector set. If the affinity is superior to a predefined value, then a non-proper element is identified.

The censor-phase of the NSA primarily consists of generating a detector set from the data that were randomly chosen and verifying which data can recognise a non-proper pattern. The detectors are similar to mature $\mathrm{T}$ cells, which can recognise pathogenic agents [20].

The monitoring phase consists of monitoring a system to identify a change in the behaviour; thus, this phase classifies the change using the detector set that was created in the censor-phase. The censor-phase occurs offline, and the monitoring-phase occurs in real time $[16,20]$.

The antigen $(\mathrm{Ag})$ is the signal to be analysed in the NSA and can be represented by expression (1). The detectors represent the antibodies $(\mathrm{Ab})$ and are expressed according to expression (2) [14, 19]

$$
\begin{aligned}
& \mathrm{Ag}=\mathrm{Ag}_{1}, \mathrm{Ag}_{2}, \mathrm{Ag}_{3}, \mathrm{Ag}_{4}, \ldots, \mathrm{Ag}_{L} \\
& \mathrm{Ab}=\mathrm{Ab}_{1}, \mathrm{Ab}_{2}, \mathrm{Ab}_{3}, \mathrm{Ab}_{4}, \ldots, \mathrm{Ab}_{L}
\end{aligned}
$$

where $L$ is the dimension of the space of the antigen and the antibody.

\subsection{Matching criterion}

To evaluate the affinity with the chains and to prove that they are similar, the matching criterion is used, which has the same meaning as the combination. The matching can be perfect or partial [21].

The matching is perfect when the two analysed chains have the same value in every position, and the matching is partial when the patterns have only one identical position value to confirm the matching, which is previously defined $[14,15]$. This quantity is known as the affinity rate. The affinity rate represents that there is a similar grade for matching to occur between two analysed chains [20]. Bradley and Tyrrell [21] defines the affinity rate according to the following equation

$$
T_{\mathrm{Af}}=\left(\frac{\mathrm{An}}{\mathrm{At}}\right) * 100
$$

where $T_{\mathrm{Af}}$ is the affinity rate; An is the quantity of normal rates in the problem (proper rates); and At is the total number of chains in the problem (proper and non-proper chains).

Equation (3) allows the precise calculation of the affinity rate for the proposed problem and represents the statistical analysis with the samples of the problem.

To dynamically improve the diagnosis, a deflection is proposed that is attached to the antibody (detector pattern-Ab), that is, a tolerance with which it is possible to accept the combination with the patterns. This tolerance is defined according to (4) [17]. This deflection acts individually in each position $i$ of vector (Ab), allowing verification of the matching in each position

$$
\underline{\mathrm{Ab}}_{i} \leq \mathrm{Ag}_{i} \leq \overline{\mathrm{Ab}}_{i}
$$

where $\mathrm{Ag}_{i}$ is the nominal value of position $i$ of the antigen (pattern under analysis); $A \mathrm{~b}_{i}$ is the nominal value of position $i$ except for the deflection adopted at the antibody (detector pattern); and $\overline{\mathrm{Ab}}_{i}$ is the nominal value of position $i$ plus the deflection adopted at the antibody (detector pattern).

In this way, if the value of position $i$ of antigen (Ag) is in the interval expressed in (4), then the position is considered to match. Thus, it is possible to quantify the affinity using the patterns, analysing position-by-position (point-by-point).

Expression (5) represents the method for quantifying the total affinity with the analysed patterns [21]

$$
\mathrm{Af}_{T}=\frac{\sum_{i=1}^{L} \mathrm{Pc}_{i}}{L} * 100
$$

where $\mathrm{Af}_{T}$ is the $\%$ of the affinity with the patterns analysed; $L$ is the total quantity of positions; $\mathrm{Pc}$ is the matched position; and $\sum_{i=1}^{L} \mathrm{Pc}$ is the sum (quantity) of the matched positions.

Thus, if $\mathrm{Af}_{T}$ is greater than $T_{\mathrm{Af}}$, then the combination/matching with the patterns occurs, that is, the patterns are considered to be equal/similar. Otherwise, there is no matching with the patterns.

\section{Wavelet transform}

The wavelet functions are mathematical transforms that can decompose the functions, which allows these functions to be re-written in more detail, that is, with a global vision. Thus, it is possible to differentiate the local characteristics of a signal with different sizes (resolutions) and to analyse all of the signals by translations. Owing to most of the wavelets have compact support, they are useful in analysing non-stationary signals. In this way, the wavelet analysis is better than the Fourier analysis [22].

There are several wavelet families. This work considers the orthonormal family functions and the Daubechies discrete family [23] because of having faster computational algorithms [22].

\subsection{Discrete wavelet transform}

Define a signal $\boldsymbol{y}[t]=\left(y_{0}, \ldots, y_{n-1}, y_{n}\right)$, which represents a discrete vector; then, it can be represented by a wavelet series, as follows [22]

$$
\boldsymbol{y}[t]=\sum_{k=0}^{N_{J}} c_{J, k} \phi_{J, k}(t)+\sum_{j=J}^{1} \sum_{l=0}^{N_{j}} d_{j, k} \boldsymbol{v}_{j, k}(t), \quad \nabla t \in\left[0, N_{0}\right]
$$




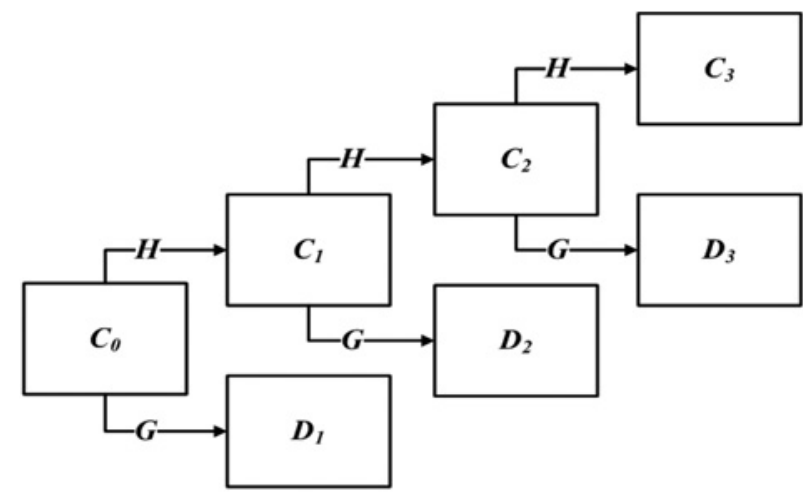

Fig. 1 Flowchart of the algorithm for DWT

where $J$ represents the resolution level, $N_{j}=(N / 2)-1$ represents the quantity of points in each new vector obtained by transformation; $\phi_{j}$, ${ }_{k}(t)$ and $v_{j, k}(t)$ are the wavelet and scale functions that perform the transformation; $j$ is the scale (dilation); and $k$ is the position (translation).

The DWT, when applied directly to a signal to generate a set of coefficients, is calculated by several entrances into a $G$ filter (low pass) and $H$ filter (high pass), which are known as resolution levels. The filters $G$ and $H$ are vectors that have constants that are already calculated and that provide an orthogonal base that is related to the scale and wavelet functions, respectively. This process is known as the Mallat Pyramidal algorithm [22] and is shown in Fig. 1.

In Fig. $1, C_{0}$ corresponds to the original discrete signal $\left(C_{0}=\boldsymbol{y}[t]\right)$, and $H$ and $G$ represent the low-pass and high-pass filters, respectively. The parameters $d_{1}, d_{2}$ and $d_{3}$ are the wavelet coefficients or the details at each resolution level and $C_{3}$ is the scale coefficients or approximations at the last level of the transform. These coefficients are obtained by a convolution of the constants with the filters by the following equations [22]

$$
\begin{aligned}
C_{j+1, k} & =\sum_{l=0}^{D-1} h_{l} C_{j, 2 k+l} \\
d_{j+1, k} & =\sum_{l=0}^{D-1} g_{l} C_{j, 2 k+l}
\end{aligned}
$$

where $k=\left[0, \ldots,\left(N / 2^{j}\right)-1\right]$ and $D$ is the number of constants in the filter. Thus, the coefficient $C_{j, k}$ represents the average local media, and the wavelet coefficient $d_{j, k}$ represents the complementary information or the details that depart from the average media. Therefore, the transform coefficients, when ordered by scale $(j)$ and position $(k)$, are represented as follows [22]

$$
\psi=\left(\left(C_{J, k}\right)_{k=0}^{N_{J}},\left(\left(d_{j, k}\right)_{k=0}^{N_{J}}\right)_{j=J}^{1}\right)
$$

such that $\psi$ is a finite representation in terms of the coefficients of the signal decomposition in (6). Fig. 2 shows the decomposition process of a signal at two resolution levels. Observe that at each transformation level, the size of the vectors is reduced by half $(N /$

\begin{tabular}{|l|l|l|ll|}
\hline$y_{0} \ldots$ & & & \\
\hline$C_{1,0} \ldots$ & & & & \\
\hline
\end{tabular}

Fig. 2 Pyramidal algorithm for DWT
Table 1 Quantity of simulations executed

\begin{tabular}{lcc}
\hline Voltage disturbance & \multicolumn{2}{c}{ System } \\
\cline { 2 - 3 } & 84 -bus & 134-bus \\
\hline outage & 48 & 48 \\
harmonic & 144 & 144 \\
swell & 72 & 72 \\
sag & 72 & 72 \\
swell with harmonic & 96 & 96 \\
sag with harmonic & 96 & 96 \\
oscillatory transient & 96 & 96 \\
total & 624 & 624 \\
\hline
\end{tabular}

$2^{J}$ ). This figure represents an adaptation of the figure that represents the pyramidal algorithm for DWT.

\section{Modelling and simulation}

In this section is presented the modelling and simulation performed in this work. Was necessary perform modelling and simulation because of the absence of a set of real data provided by any distribution company. So, in the paper aided by model routines [24] in EMTP software [25], two electrical distribution systems were modelled, which were a test system with 84-bus and a real system with 134-bus. The sample frequency is $15.36 \mathrm{kHz}$, which corresponds to 256 samples per cycle. The simulation time is 200 ms.

In this paper, it is simulated the voltage disturbances using the theoretical model proposed by Abdel-Galil et al. [26]. With this model, each disturbance is generated by varying the parameters in the equations that represent the wave form of the disturbance (e.g. outage, swell, sag etc.). This type of disturbance is introduced on EMTP simulator [25] using the routine models [24].

A total of 1248 simulations was performed, with 624 for each electrical system. The simulations consider the phase in which the disturbance occurs, the loadings of $50-120 \%$ and the parameters of the theoretical model. Table 1 presents the quantity of simulations that are executed for each disturbance class.

\section{Proposed methodology}

The wavelet immune algorithm proposed in this work to detect and classify voltage disturbances is based on the negative selection principle, and the phases are presented as follows.

\subsection{Censor-phase}

This phase generates the proper detectors and the disturbance detector set. The detector sets are used by the diagnosis system during the monitoring process. The detectors are generated for each electrical system phase.

The proper detectors represent the normal operation characteristics of the electrical system; therefore, they are placed in oscillograph windows (256 points) that represent the normal operation. Once a proper detector is generated, it is possible to generate the disturbance detectors. This process is illustrated in Fig. 3.

Next, the procedure is divided into three modules: the reading of the signals to create the detectors, the wavelet module that decomposes the signals using a DWT with four resolution levels and the censor module with which the signals are randomly chosen and that verify the matching in relation to the proper detector set. If the affinity criterion is satisfied, the signals are rejected because they have proper characteristics. Otherwise, the signals are placed in the disturbance detector set.

The quantity of detectors that are used is determined by the operator. However, it is recommended to use $30 \%$ of the available data. The matching criterion is proposed in [21], which uses a deviate of $3 \%$. 


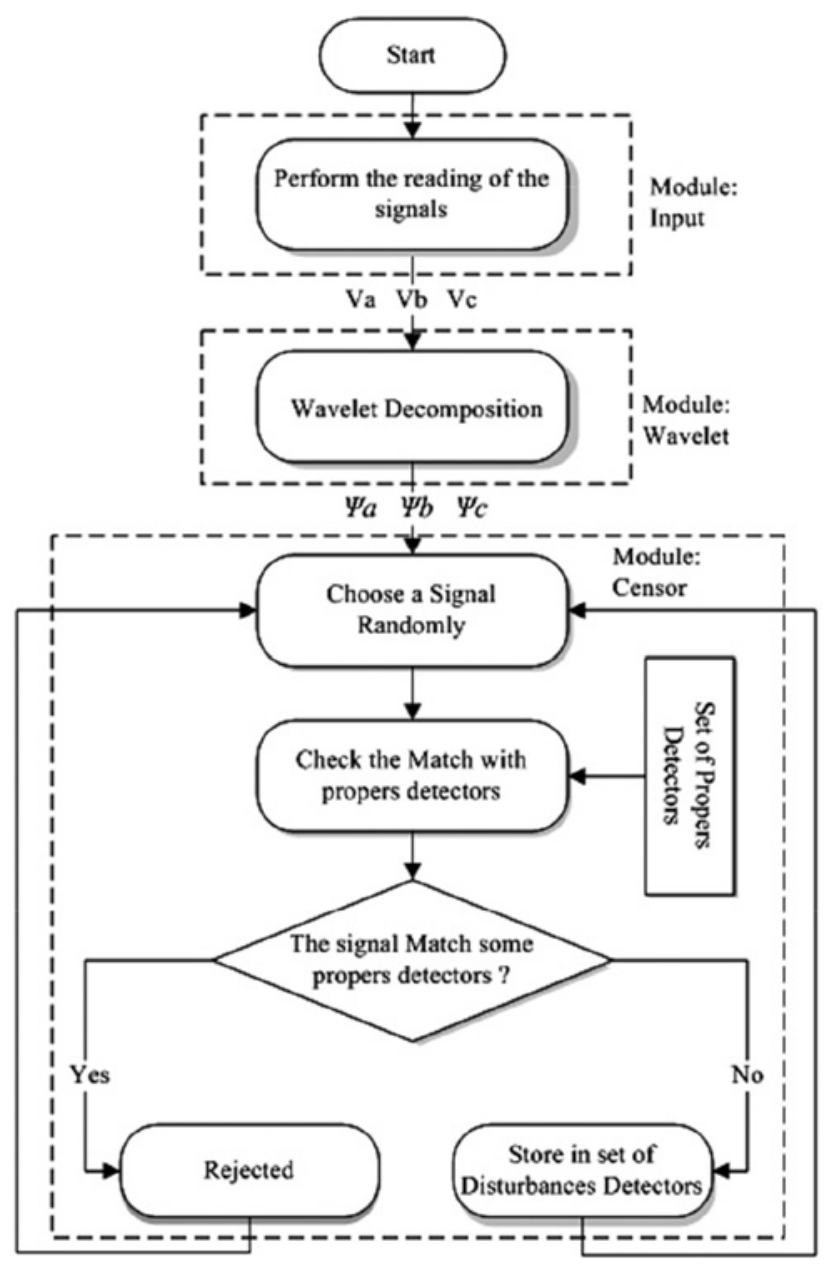

Fig. 3 Flowchart of the censor-phase

\subsection{Monitoring-phase}

The monitoring-phase is divided into five modules: the input or the reading of the signals (by the SCADA acquisition data system [27]), the wavelet module that decomposes the signals into four resolution levels, the detector module, which performs the discrimination of proper/non-proper, and the extraction module, which extracts the non-proper signals that are detected and, finally, the classification module to classify the disturbances. Fig. 4 illustrates the monitoring-phase.

The wavelet module is executed after the signal is acquired and decomposes the signals, transforming the signals to the wavelet domain. Afterward, the detector module compares the signals that are under analysis with the proper detectors to identify the match with the signals. This module performs the diagnosis of the analysed signals into proper and non-proper categories.

When an abnormality is detected, the abnormal signal is extracted and the classification module is executed. The classification module compares the abnormal signal to the disturbance detector set, and the matching is verified. Thus, the abnormal signal is classified according to the detector class that the signal matches.

This phase uses the partial matching criterion proposed in [21], adopting a deviation of $3 \%$ in the detectors.

\subsection{Wavelet decomposition module}

The wavelet decomposition module is important to extract and emphasise the signal characteristics, which are easily detected in the wavelet world.

In this paper, were used four levels of decomposition for the DWT. It was decided to use four levels of decomposition, because with an approximation of four levels in the components of the DWT, the abnormalities in the signals are presented more easily. Table 2 is presented the frequency ranges for each level of resolution in the DWT used in this paper.

Fig. 5 illustrates a signal that has a transient oscillatory disturbance in phase A. This signal is presented at the input of the wavelet decomposition module. After processing the signal, it appears according to Fig. 6.

These figures show the importance of wavelet decomposition for the diagnosis system. The abnormality at phase $\mathrm{A}$ is emphasised when the signal is decomposed in the wavelet world. Thus, the
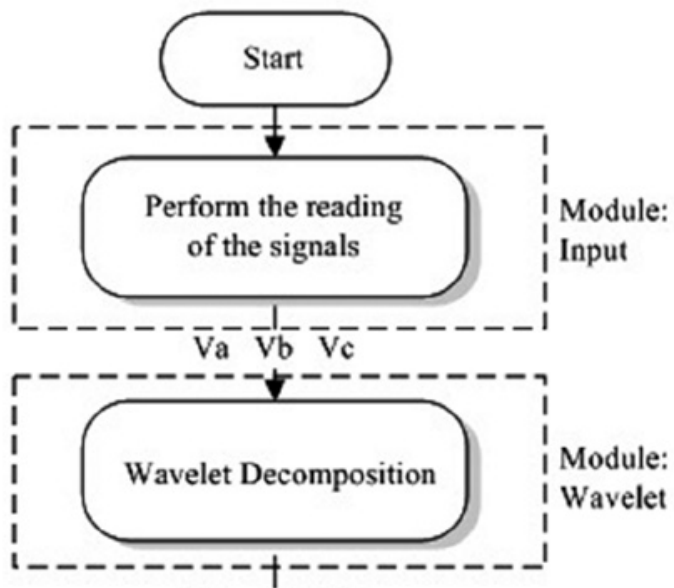

$\Psi_{a} \quad \Psi_{b} \quad \Psi_{c}$

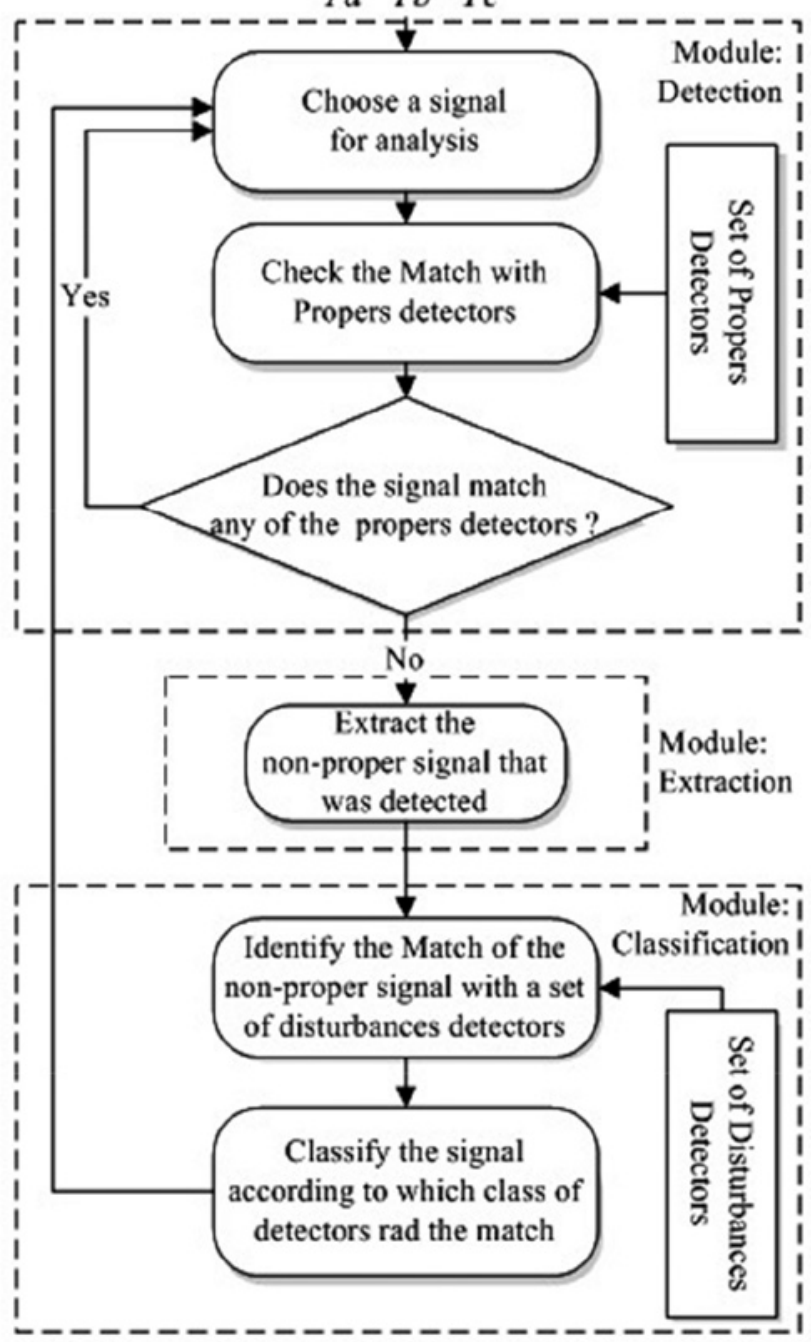

Fig. 4 Processing steps of the example 
Table 2 Frequency ranges for each level of resolution in the DWT

\begin{tabular}{lcc}
\hline Resolution levels & Parameter & Frequency range, $\mathrm{kHz}$ \\
\hline 1 & $D_{1}$ component & $7.68-3.84$ \\
2 & $D_{2}$ component & $3.84-1.92$ \\
3 & $D_{3}$ component & $1.92-0.96$ \\
4 & $D_{4}$ component & $0.96-0.48$ \\
4 & $C_{4}$ component & $0.00-0.48$ \\
\hline
\end{tabular}

wavelet module contributes to the NSA because it is sensitive when analysing patterns and allows for easy recognition of any abnormality.

\section{Results}

This section presents the results that are obtained with the proposed method with the test systems. Every simulation is executed with a PC Intel Core 2 Duo $1.9 \mathrm{GHz}, 2$ GB of Memory RAM (Random-Access Memory), and an operational system Windows 7 Ultimate 32 bits. The algorithm was developed in MATLAB ${ }^{\mathbb{B}}$ [28]. The proposed algorithm is applied to the distribution systems that have 84 and 134-buses [29, 30].

\subsection{4-bus system}

The 84-bus system is a test system that has 83-bus of loads, one substation and 83 circuits. The base voltage is $11.4 \mathrm{kV}$. The conditions of the active and reactive loads are $28.350 \mathrm{~kW}$ and $20.700 \mathrm{kVAr}$, respectively [29]. Fig. 7 shows the topology of the system.

Table 3 presents the results that were obtained with the systems of voltage disturbance diagnosis for the 84-bus system [29].

\subsection{Real system of 134 -bus}

This system is a real distribution system that has the following characteristics: aerial, three phase, several branches, composed of 134-bus, $13.8 \mathrm{kV}, 7.065 \mathrm{MVA}$, mutually coupled and the power factor is equal to 0.92 [30]. This electrical system represents part of the distribution system of the hydroelectric power plant Jupiá in Três Lagoas city, São Paulo, Brazil. Fig. 8 shows the topology of the system.

Table 4 presents the results that were obtained with the systems of voltage disturbances diagnosis for the 134-bus system [30].

The results show the correct percentage in identifying the voltage disturbances in relation to the quantity of patterns used on the tests. It should be noted especially that the WAIS is executed in $<90 \mathrm{~ms}$.

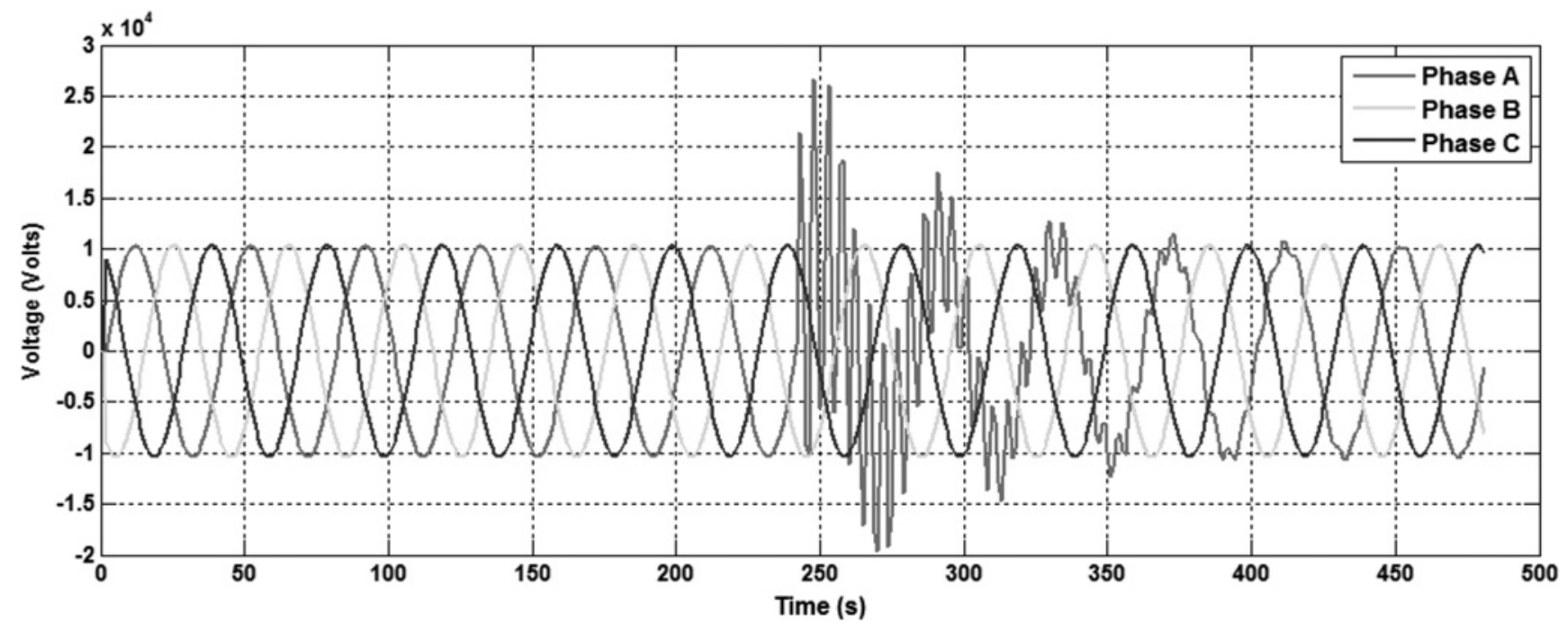

Fig. 5 Signal with a transient oscillatory disturbance at phase A

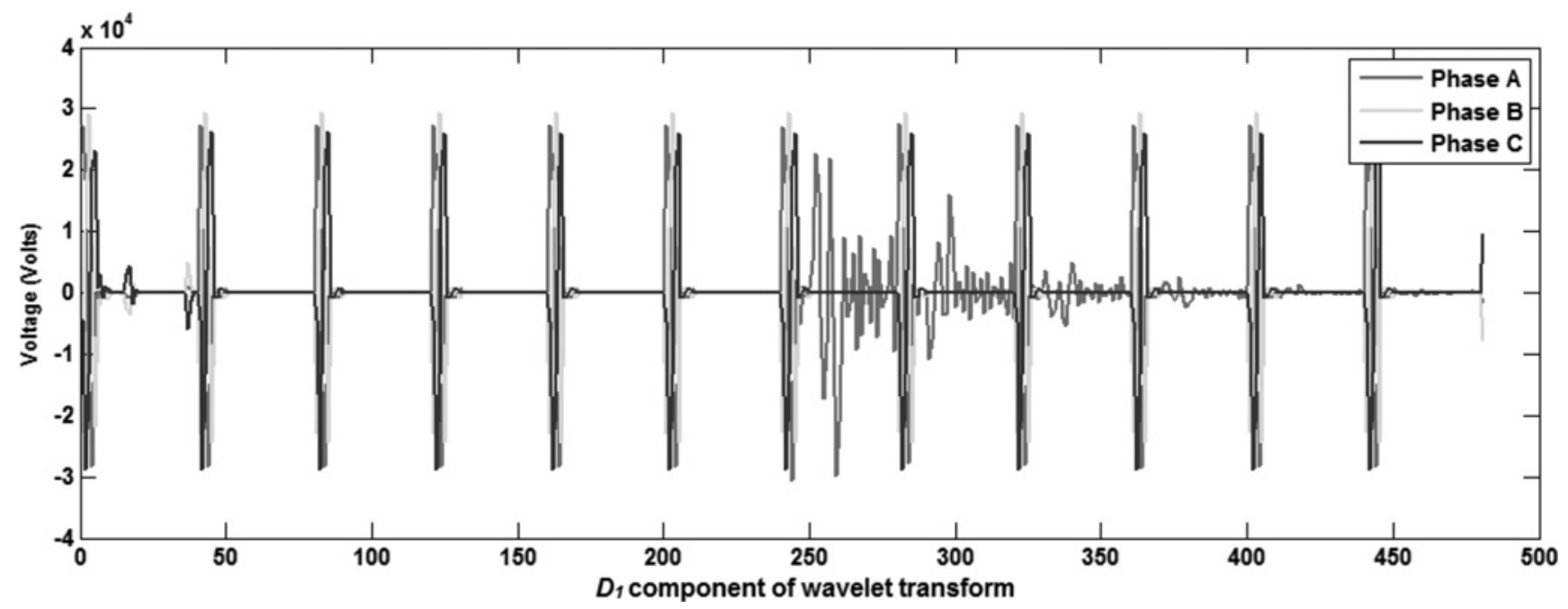

Fig. 6 Wavelet decomposition signal 

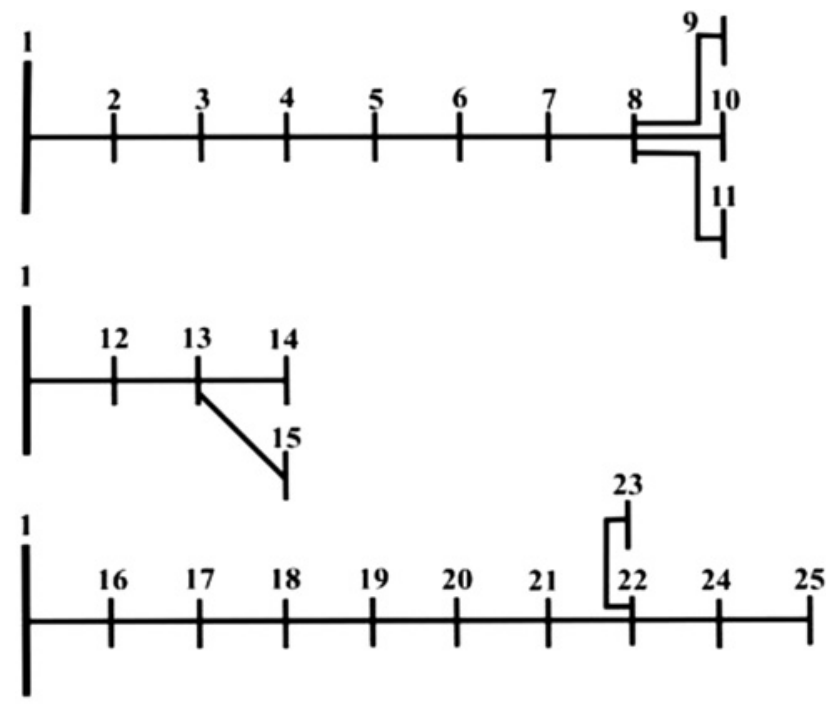

1
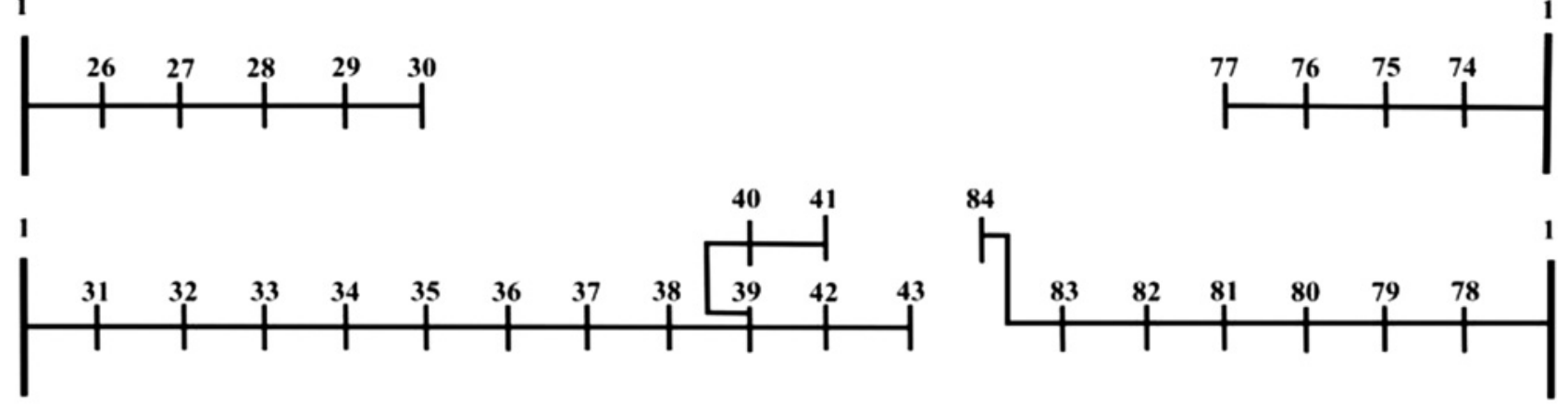

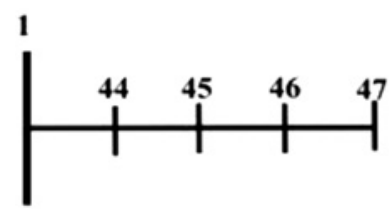

Fig. 7 System of 84-bus line flow

With the introduction of the wavelet module in the method, was obtained an easy identification of the abnormalities, because when in the wavelet domain the detailed coefficients of the signals are disarranged, which indicates the presence of abnormalities in the signal.

\subsection{Comparative study}

Table 5 presents a comparative study that uses matching according to the proposed method and the principal references that are available in the literature.

The proposed method presents a matching index and a precision that is superior to other similar works that are available in the

Table 3 Results for the 84-bus system

\begin{tabular}{lcc}
\hline Diagnosis & \multicolumn{2}{c}{ 84-bus } \\
\cline { 2 - 3 } & Tested patterns & Match, \% \\
\hline swell & 72 & 100.00 \\
sag & 72 & 100.00 \\
outage & 48 & 100.00 \\
harmonic & 144 & 100.00 \\
swell with harmonic & 96 & 100.00 \\
sag with harmonic & 96 & 100.00 \\
oscillatory transient & 96 & 100.00 \\
total & 624 & 100.00 \\
\hline
\end{tabular}

literature. It should be emphasised that the use of the wavelet transform changes the sensitivity of the voltage disturbance diagnosis and can detect any abnormality; therefore good results are presented.

Although the results present an accuracy of $100 \%$ for simulated cases in EMTP, with real data probably the result would be different (with less accuracy). However, the methodology proposed in this paper could be implemented in practice, providing efficiency and robustness for the system.

\section{Conclusions}

This paper presents a new approach to detect and classify voltage disturbances in electrical distribution systems using WAIS algorithm. The EMTP software is used to simulate the disturbances, generating a data set. The proposed algorithm presents good results, with matches very close to $100 \%$ in detecting and classifying the abnormalities tested. The generating detector phase is executed offline with no prejudice for the algorithm. The monitoring-phase quickly executes in a total time of $<90 \mathrm{~ms}$, which allows for it to be used in real time to aid the decision making. The combination of the wavelet transform with the NSA provides more precision to the diagnosis because of the high resolution level in decomposing signals, making it easy to identify the abnormalities. Thus, the wavelet immune algorithm 


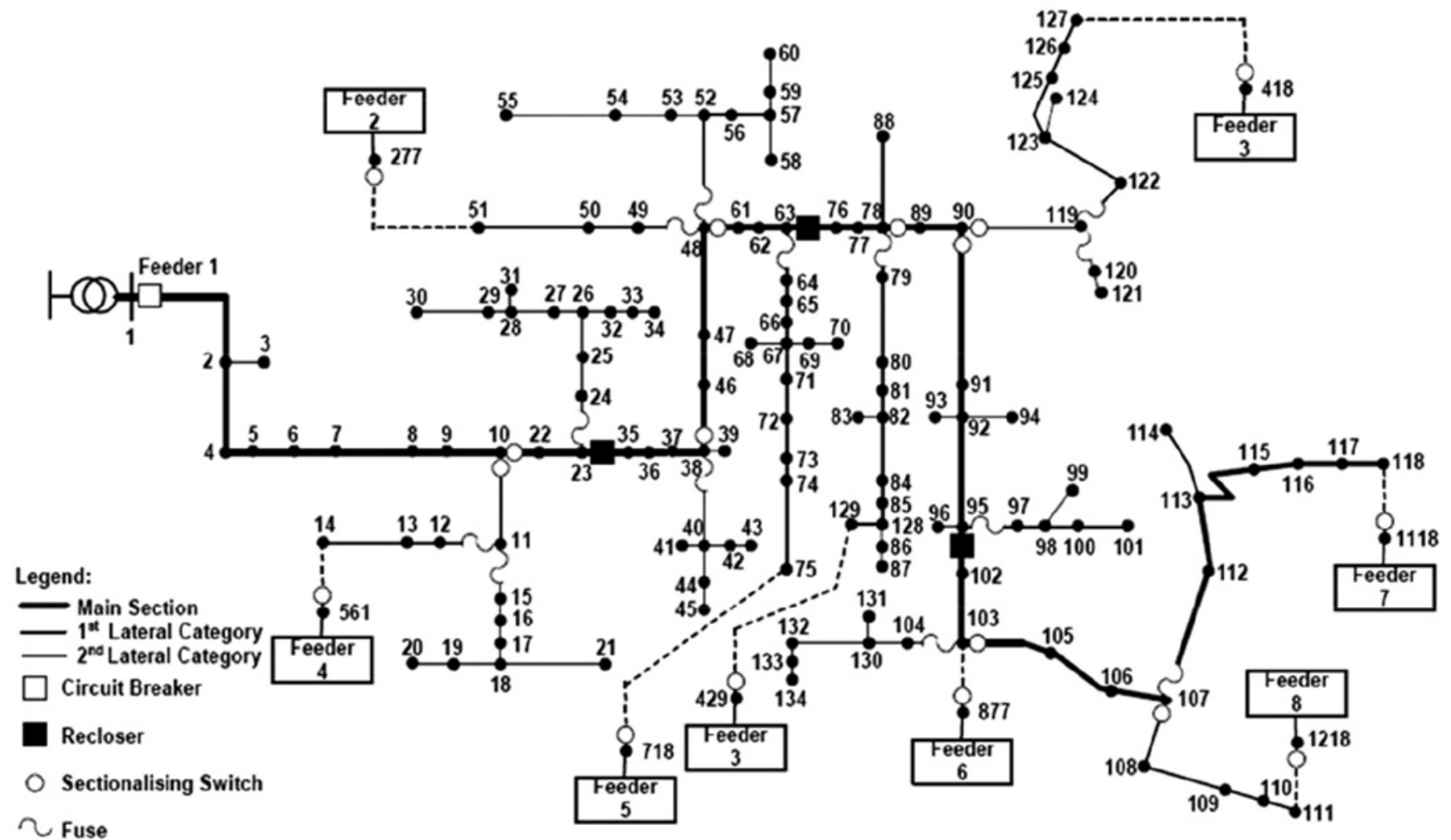

Fig. 8 Real system of 134-bus line flow

Table 4 Results for the 134-bus system

\begin{tabular}{lcc}
\hline Diagnosis & \multicolumn{2}{c}{ 134-bus } \\
\cline { 2 - 3 } & Tested patterns & Match, \% \\
\hline swell & 72 & 100.00 \\
sag & 72 & 100.00 \\
outage & 48 & 100.00 \\
harmonic & 144 & 100.00 \\
swell with harmonic & 96 & 100.00 \\
sag with harmonic & 96 & 100.00 \\
oscillatory transient & 96 & 100.00 \\
total & 624 & 100.00 \\
\hline
\end{tabular}

Table 5 Comparative study

\begin{tabular}{lccc}
\hline References & $\begin{array}{c}\text { Electrical system/ } \\
\text { theoretical model }\end{array}$ & Software used & $\begin{array}{c}\text { Total } \\
\text { match, \% }\end{array}$ \\
\hline$[8]$ & $\begin{array}{c}{[26]} \\
{[12]}\end{array}$ & MATLAB & 96.21 \\
{$[14]$} & not specified & MATLAB/ & 99.00 \\
{$[10]$} & Simulink & \\
{$[13]$} & 134 -bus & ATP/EMTP & 99.11 \\
{$[17]$} & $134-$-bus & ATP & 99.31 \\
this work & $134-$ bus & ATP/EMTP & 99.66 \\
this work & 84-bus & ATP/EMTP & 99.72 \\
\hline
\end{tabular}

that is proposed is precise, robust and efficient and is allowed in several applications, principally in a smart grid environment.

\section{Acknowledgments}

The authors thank CAPES-PDSE (Proc. No BEX-3659/14-3) and FAPESP (Proc. No 2011/06394-5) for supporting this study.

\section{References}

1 Mcdonald, J.D.: 'Electric power substations engineering' (CRC Press, 2003, 2nd edn.)

2 Alag, S., Agogino, A.M., Morjaria, M.: 'A methodology for intelligent sensor measurement, validation, fusion, and fault detection for equipment monitoring and diagnosis', Artif. Intell. Eng. Design Anal. Manuf., 2001, 15, (4), pp. 307-320

3 Northcote-Green, J., Wilson, R.: 'Control and automation of electrical power distribution systems' (Taylor \& Francis, 2007, 1st edn.)

4 Santoso, S., Powers, E.J., Grady, W.M., Parsons, A.C.: 'Power quality waveform recognition using wavelet-based neural classifier - Part 1: theoretical foundation', IEEE Trans. Power Deliv., 2000, 15, (1), pp. 222-228

5 Benmokhtar, R., Huet, B.: 'Neural network combining classifier based on Dempster-Shafer theory'. Proc. of the Int. Multi Conf. on Computer Science and Information Technology, 2006, pp. 3-10

$6 \mathrm{He}, \mathrm{H}$., Starzyk, J.A.: 'A self-organizing learning array system for power quality classification based on wavelet transform', IEEE Trans. Power Deliv., 2006, 21, (1), pp. 286-295

7 Reaz, M.B.I., Choong, F., Sulaiman, M.S., Mohd-Yasin, F., Kamada, M.: 'Expert system for power quality disturbance classifier', IEEE Trans. Power Deliv., 2007, 22, (3), pp. 1979-1988

8 Uyar, M., Yildririm, S., Gencoglu, M.T.: 'An effective wavelet-based feature extraction method classification of power quality disturbance signals', Electr. Power Syst. Res., 2008, 78, (10), pp. 1747-1755

9 Werbos, P.J.: 'Beyond regression: new tools for prediction and analysis in the behavioral sciences'. PhD. thesis, Harvard University, 1974

10 Oleskovicz, M., Coury, D.V., Felho, O.D., Usida, W.F., Carneiro, A.F.M., Pires, L. R.S.: 'Power quality analysis applying a hybrid methodology with wavelet transform and neural networks', Int. J. Electric. Power Energy Syst., 2009, 31, (5), pp. 206-212

11 Masoum, M.A.S., Jamali, S., Ghaffarzadeh, N.: 'Detection and classification of power quality disturbances using discrete wavelet transform and wavelet networks', IET Sci. Meas. Technol., 2010, 4, (4), pp. 193-205

12 Zhang, M., Li, K., Hu, Y.: 'A real-time classification method of power quality disturbances', Electr. Power Syst. Res., 2011, 81, (12), pp. 660-666

13 Decanini, J.G.M.S., Tonelli-Neto, M.S., Malange, F.C.V., Minussi, C.R. 'Detection and classification of voltage disturbances using a fuzzy-ARTMAP-wavelet network', Electr. Power Syst. Res., 2011, 81, (12), pp. 2057-2065

14 Lima, F.P.A., Lotufo, A.D.P., Minussi, C.R.: 'Artificial immune systems applied to voltage disturbance diagnosis in distribution electrical systems'. Proc. of PowerTech, Grenoble, France, June 2013, pp. 1-6

15 Lima, F.P.A., Lotufo, A.D.P., Minussi, C.R.: 'Disturbance detection for optimal database storage in electrical distribution systems using artificial immune systems with negative selection', Electr. Power Syst. Res., 2014, 109, pp. 54-62

16 de Castro, L.N., Timmis, J.: 'Artificial immune systems: a new computational intelligence approach' (Springer-Verlag, 2002, 1st edn.) 
17 Lima, F.P.A.: 'Analysis of voltage disturbances in distribution electrical systems based on artificial immune systems'. Master thesis, UNESP, Univ Estadual Paulista, 2013, (in Portuguese)

18 Forrest, S., Perelson, A., Allen, L., Cherukuri, R.: 'Self-nonself discrimination in a computer'. Proc. of IEEE Symp. on Research in Security and Privacy, 1994, pp. 202-212

19 de Castro, L.N.: 'Immune engineering: development and application of computational tools inspired by artificial immune systems'. $\mathrm{PhD}$ thesis, UNICAMP, 2001, (in Portuguese)

20 Dasgupta, D.: 'Artificial immune systems and their applications' (Springer, 1998, 1st edn.)

21 Bradley, D.W., Tyrrell, A.M.: 'Immunotronics - novel finite-state-machine architectures with built-in self-test using self-nonself differentiation', IEEE Trans. Evol. Comput., 2002, 6, pp. 227-238

22 Mallat, S.: 'A wavelet tour of signal processing' (Academic Press, 1999, 2nd edn.)

23 Daubechies, I.: 'Ten lectures on wavelets' (Society for Industrial and Applied Mathematics, 1992, 1st edn.)
24 Dubé, L.: 'How to use MODELS-based user-defined network components in ATP'. Proc. of EEUG, Budapest, 1996, pp. 10-12

25 EMTP-RV: 'Eletromagnetic transient program, powersys-solutions'. Le Puy-Sainte-Réparade, France, 2011

26 Abdel-Galil, T.K., Kamel, M., Youssef, A.M., El-Saadany, E.F., Salama, M.M.A.: 'Power quality disturbance classification using inductive inference approach', IEEE Trans. Power Deliv., 2004, 19, (4), pp. 1812-1818

27 Wylie, M., Pleydell, M.: 'Network management data acquisition and analysis, road transport information and control'. Proc. of RTIC, United Kingdom, 2008, pp. 1-3

28 MATLAB: '7.8 Version', Mathworks Company, 2011

29 Chiou, J.P., Chang, C.F., Su, C.T.: 'Variable scaling hybrid differential evolution for solving network reconfiguration of distribution systems', IEEE Trans. Power Systems, 2005, 20, (2), pp. 668-674

30 LAPSEE: 'Practical134-bus feeder data', Electrical Power System Planning Laboratory, 2011, Available at: http://www.dee.feis.unesp.br/lapsee/ 